\title{
Avaliação de Treino de Controle do Stress para Mães de Crianças com Transtornos do Espectro Autista
}

\author{
Stress Control Training Assessment for Mothers of Children \\ with Autism Spectrum Disorders
}

\author{
Glória de Fátima Araújo Moxotó*, ${ }^{a}$ \& Lucia Emmanoel Novaes Malagris ${ }^{b}$ \\ a Marinha do Brasil, Rio de Janeiro, RJ, Brasil \\ $\&{ }^{b}$ Universidade Federal do Rio de Janeiro, Rio de Janeiro, RJ, Brasil
}

\begin{abstract}
Resumo
O diagnóstico de transtorno do espectro autista (TEA) em filhos tem sido descrito na literatura como razão de excessivo stress em suas mães. Como o Treino de Controle do Stress (TCS) de Lipp, já foi adaptado com êxito para o atendimento de diversas populações de diferentes faixas etárias, o objetivo deste trabalho foi investigar a eficácia de uma adaptação do TCS para mães de crianças com TEA (TCS-MTEA) na redução do stress desta população. Para isto comparou-se a presença e níveis de stress de vinte participantes, na faixa etária entre 20 e 50 anos, sendo dez submetidas a esta intervenção individual configurando uma amostra experimental (AE) e outras dez que tiveram apenas níveis de stress avaliados no mesmo intervalo de tempo, configurando uma amostra controle (AC). As vinte participantes foram avaliadas antes e após o treino com o Inventário de Sintomas de Stress para Adultos de Lipp. Ao final da intervenção das dez participantes estressadas inicialmente na AE, sete estavam sem stress (70\%) e as outras três apresentaram redução do nível de stress (30\%) enquanto as dez da AC (100\%) continuavam estressadas. Conclui-se que o TCS-MTEA foi eficaz na redução do stress das participantes da $\mathrm{AE}$.

Palavras-chave: Transtornos do espectro autista, mães, treino de controle do stress.
\end{abstract}

\begin{abstract}
The diagnosis of autism spectrum disorder (ASD) in a child has been described in literature as associated to excessive stress in their mothers. As the Lipp Stress Control Training (referred to locally as TCS) has been successfully adapted for the care of diverse populations of different ages, the aim of this study was to investigate the effectiveness of an adaptation of TCS for mothers of children with ASD (referred to locally as TCS-MTEA) in reducing stress in this population. For this, the presence and levels of stress of twenty participants aged between 20 and 50 years was compared. Ten individuals were submitted to individual training, configuring an experimental sample (ES) and ten only had stress levels evaluated in the same time interval, configuring a control sample (CS). The twenty participants were assessed before and after training with the Lipp Stress Symptoms Inventory. After the intervention, among the ten ES participants initially stressed, seven were without stress $(70 \%)$ and the other three had the level of stress reduced (30\%) while the ten CS participants $(100 \%)$ remained stressed. The study concluded that the TCS-MTEA was effective in reducing the stress of the ES participants.

Keywords: Autism spectrum disorders, mothers, stress management training.
\end{abstract}

O stress emocional tem sido estudado por pesquisadores nacionais e internacionais em diferentes contextos da vida humana: social, saúde, trabalho e família (Lipp \& Malagris, 2001, 2011). Considerando que estas diferentes áreas são interligadas e exercem efeitos sistêmicos umas sobre as outras, compreende-se que o stress excessivo em

\footnotetext{
" Endereço para correspondência: Avenida Pasteur, 250, Praia Vermelha, Rio de Janeiro, RJ, Brasil 22290-240. E-mail: gloriamoxoto@gmail.com

Agradecemos à Dra. Marilda Lipp, por ter autorizado a adaptação do treino de controle do stress que tem trazido benefícios a tantas vidas e pelas valiosas sugestões no exame de qualificação do projeto de pesquisa.
}

qualquer uma delas ocasionará impactos nas demais. Por ser a família, de uma forma geral, o principal núcleo afetivo de um indivíduo, o stress excessivo neste contexto se destaca dos outros, pela decorrente capacidade de afetar o equilíbrio individual, disseminando o impacto para outras áreas de sua vida (Lipp \& Malagris, 2001, 2011).

No contexto familiar, o stress parental configura-se de maneira acentuadamente deletéria, uma vez que o curso favorável do desenvolvimento humano é influenciado pela qualidade das interações parentais e familiares (Carter \& McGoldrick, 1995; Szymanski, 2004). Assim, diversos autores têm descrito a influência do stress excessivo na potencialização de conflitos e exacerbação de problemas de 
comportamento agravando situações onde há dificuldades de relacionamento entre pais e filhos (Kazdin \& Whitley, 2003). Especificamente na relação parental, a presença de patologia crônica em um filho na infância costuma potencializar a probabilidade de surgimento de intenso stress (Bradford, 1997; Castro \& Piccinini, 2002). Ao lado de outros fatores, a necessidade de reorganização da estrutura parental prévia ao surgimento da doença, para atender o novo conjunto de demandas suscitadas em termos de convívio e tratamento pode se configurar num potente estressor (Bradford, 1997). Desta maneira, além do ajuste na expectativa de ter um filho saudável, os pais de filhos cronicamente enfermos têm que adequar suas rotinas ao atendimento das exigências diárias de uma situação de doença permanente (Bradford, 1997).

O impacto de diversas doenças crônicas de filhos na relação parental tem sido estudado por intermédio da avaliação dos níveis de stress dos pais e mães. Assim várias pesquisas têm sido conduzidas com o objetivo de investigar o stress parental em filhos com doenças renais, transtorno do déficit de atenção e hiperatividade (TDAH), síndrome de Down, paralisia cerebral e transtornos do espectro autista (Bellé, Andreazza, Ruschel, \& Bosa, 2009; Fávero \& Santos, 2005; Hayes \& Watson, 2012; Schmidt \& Bosa, 2007).

Dentre as patologias citadas, o diagnóstico de transtorno do espectro autista (TEA) em um filho tem sido descrito por diversos autores como razão de excessivo stress parental, seja em comparação ao stress de pais de filhos com desenvolvimento típico, ou de filhos com outras doenças (Cadman et al., 2012; Hayes \& Watson, 2012). Cabe ressaltar que, comparativamente em relação aos pais, as mães apresentam níveis de stress significativamente mais elevados nesta população (Bendixen et al., 2011; Dabrowska, \& Pisula, 2010; Schmidt \& Bosa, 2007; Sifuentes \& Bosa, 2010). Estas mães, mesmo quando avaliadas em diferentes contextos culturais, apresentam níveis intensos de stress emocional (Bilgin \& Kucuk, 2010; Konstantareas \& Papageorgiou, 2006; Phetrasuwan $\&$ Shandor Miles, 2009).

As características dos TEA e suas interfaces com os aspectos relacionais da díade mãe e filho podem auxiliar o entendimento da magnitude dos níveis de stress destas mães. Os TEA se caracterizam pela presença de uma tríade de prejuízos nas áreas de interação social, comunicação e repertório de comportamentos estereotipados e repetitivos (Tonge \& Brereton, 2011). Desta forma, as interações sociais da criança com TEA têm como característica a escassez de reciprocidade e espontaneidade. Embora estas crianças possam apresentar resposta aos estímulos de seus pares, esporadicamente tomam a iniciativa de começar a interação ou conseguem estabelecer sua manutenção (Sanini, Brum, \& Bosa, 2010). Em decorrência deste padrão interacional falho, bem como da dificuldade na comunicação de seus desejos, as mães costumam experimentar constante sentimento de frustração (Sanini et al., 2010). De acordo com Malagris (2009) este sentimento pode originar stress crônico, se muito presente na vida de um indivíduo.

Além dos sentimentos de frustração geralmente experimentados por estas mães, muitos são os fatores associados à preponderância do stress materno em relação ao paterno dentre os pais de indivíduos com TEA. Pode ser citado como fator associado à preponderância do stress materno em relação ao paterno dentre os pais de indivíduos com TEA, o fato de serem as mães as principais cuidadoras de seus filhos com TEA, sendo elas responsáveis por eles em casa, e nos tratamentos realizados (Bosa, 2002; Moes, Koegel, Schreibman, \& Loos, 1992; Schmidt, 2004). Adicionalmente, os cuidados infantis são atribuídos à mãe pela sociedade e ainda que o papel materno não seja o único exercido, as expectativas sociais sobre o desempenho dos filhos recaem sobre ela (Navarro, 2007). Tamanha responsabilidade pode atuar como mais um estressor para a mãe, se o desenvolvimento e o desempenho do filho não estão consonantes com as normas sociais, como no caso de uma criança com TEA. Finalmente, o luto pela perda do filho imaginado, por ocasião do diagnóstico de TEA, pode também atuar como forte estressor para as mães (Sanini et al., 2010).

Como agravante, também são muitos os prejuízos ocasionados a estas mulheres em decorrência da magnitude do stress vivenciado por elas. Quanto às consequências do stress excessivo destas mães aparece associado ao surgimento de problemas de saúde física e mental (Hastings et al., 2005; Lovell, Moss, \& Wetherell, 2012), prejuízos financeiros e laborativos (Cidav, Marcus, \& Mandell, 2012), relacionamento dificultado com os cônjuges e demais filhos (Gomes \& Bosa, 2004; Sifuentes \& Bosa, 2010), aumento no número de divórcios (Hartley et al., 2010) e, finalmente, impacto negativo no tratamento de seus filhos (Osborne, McHugh, Saunders, \& Reed, 2008).

Diante de tão expressivo quadro de danos diversos, vários pesquisadores sugerem o planejamento de programas psicoeducacionais para mães de crianças com TEA, focados no manejo do stress e das emoções, modificação de crenças disfuncionais e resolução de problemas diários ligados ao diagnóstico dos TEA, baseados na terapia cognitivo-comportamental e em programas de manejo do stress. Os autores propõem ainda, tratamentos que proporcionem às mães de crianças com TEA, informação acerca de sua própria saúde e necessidade de engajamento em atividades saudáveis, bem como psicoeducação que possibilite às mães lidar com as necessidades de seu filho com o objetivo de contribuir para o bem estar e para a saúde mental materna (Bourke-Taylor, Pallant, Law, \& Howie, 2012; Cappe, Wolff, Bobet, \& Adrien, 2011).

Desta forma, configura-se a relevância de disponibilizar recursos para o manejo do stress excessivo destas mães. De acordo com Lipp e Malagris (2011) a abordagem psicoterapêutica cognitivo-comportamental para o controle do stress possibilita não só a reestruturação cognitiva, ou seja, uma forma mais realista e adaptativa de entender as situações vivenciadas, como também ampliação do repertório 
comportamental e emocional para o manejo funcional dos estressores. Em concordância à proposição das autoras, o manejo psicológico do stress excessivo tem sido testado, em diversas populações clínicas e não clínicas, há décadas, em pesquisas internacionais (Antoni et al., 2009; Bougea, Darviri, \& Alexopoulos, 2011; Keypour, Arman, \& Maracy, 2011; Lutgendorf et al., 1998; Steinmetz, Kaplan, \& Miller, 1982). No Brasil, diferentes intervenções com populações diversas de variadas faixas etárias vêm sendo conduzidas por vários autores que obtêm êxito utilizando o Treino de Controle do Stress (TCS) de Lipp (1984; Lipp \& Malagris, 2011). O TCS foi adaptado para pessoas com hipertensão (Lipp \& Malagris, 2011), para crianças (Bignotto, 2010) e para relações interpessoais no trabalho (Sadir, 2010), com o objetivo de atender às especificidades características destas populações.

O TCS é um tratamento de base cognitivo-comportamental que se propõe a ensinar ao indivíduo maneiras de manejar melhor o stress, com o objetivo de impedir que atinja níveis excessivos, provocando efeitos nocivos a qualidade de vida em todas as áreas (Lipp \& Malagris, 2001, 2011). Esta intervenção foi concebida com base no treino de inoculação do stress de Meichenbaun (1985), na terapia racional-emotiva de Ellis (1973) e em informações derivadas de anos de pesquisa clínica com os temas de stress e hábitos de vida potencialmente estressantes conduzida na Pontifícia Universidade Católica (PUC) de Campinas por Lipp (1996).

Este tratamento tem caráter focal breve, com no máximo quinze sessões que têm início com uma análise funcional das fontes internas e externas de stress do indivíduo. Os fatores principais de ação do TCS são o reconhecimento dos estressores internos e externos, aprendizagem de formas de lidar com os mesmos ou, por intermédio da reestruturação cognitiva, o desenvolvimento de uma visão mais adaptativa sobre os fatos (Lipp \& Malagris, 2001, 2011). O TCS tem como metas, modificações de hábitos de vida e de padrões comportamentais potencialmente deletérios, em quatro dimensões que se configuram como as bases do TCS: atividade física, nutrição adequada e anti-stress, treinos de relaxamento tanto físicos quanto mentais, e modificações em padrões cognitivos e comportamentais (Lipp \& Malagris, 2001, 2011).

O objetivo de adaptar o TCS para mães de crianças com TEA foi o de preservar as bases deste tratamento, complementando-o para atender às necessidades desta população específica. Assim, a adaptação manteve os pilares do TCS e acrescentou: psicoeducação sobre TEA, explicações sobre o modelo duplo ABC-X de adaptação familiar (McCubbin \& Patterson, 1983), treino de respiração como relaxamento e atividades físicas adaptadas à rotina peculiar destas mães, treino de comunicação, crenças irracionais correlacionadas a comportamentos do filho, além de ênfase no manejo de sintomas de ansiedade e depressão.

Os tratamentos encontrados na literatura para mães de crianças com TEA são os grupos de orientação de pais, de grande valor para prover informações e ampliar o suporte social. O foco destes grupos, porém, são as crianças, suas características e como lidar com elas. $\mathrm{O}$ TCS adaptado poderia promover além das informações necessárias às mães sobre suas crianças, uma abordagem específica às características subjetivas de cada mãe. Um tratamento que considera estes aspectos pode promover o empoderamento destas mães. De acordo com Cortez e Souza (2008) o empoderamento feminino abrange duas dimensões: coletiva e individual. Assim, segundo os autores, o empoderamento se refere à identificação de restrições sociais às quais as mulheres estão submetidas e a necessidade de prover recursos para reverter esta situação, promovendo modificações em contextos amplos e públicos, como serviços de saúde adequados e em contextos específicos ou individuais, como elevação da auto-estima, aumento da autonomia e reformulação da organização do trabalho doméstico etc.

Desta maneira, a adaptação do TCS poderia propiciar mudanças nas duas dimensões: coletiva e individual. Coletiva, por se configurar como oferta de um tratamento de saúde que leva em consideração as necessidades do papel materno destas mulheres e individual, uma vez que pretende aumentar a auto-eficácia, auto-estima e organização das tarefas concernentes à maternagem de crianças com TEA (Cortez \& Souza, 2008).

Pretendeu-se, portanto, neste estudo, com base nas bem sucedidas adaptações a diversas populações, descritas na literatura, adaptar o TCS com o objetivo de contemplar as necessidades das mães de crianças com TEA. Assim, foi investigada a eficácia do "Treino de controle do stress para mães de crianças com Transtornos do Espectro Autista" a partir da comparação com uma amostra controle.

\section{Método}

Participaram do estudo 20 mães de crianças com Transtornos do Espectro Autista, atendidas em serviço interdisciplinar de atendimento ambulatorial composto por médicos, fonoaudiólogas, psicólogas, fisioterapeutas e terapeutas ocupacionais. Os critérios de inclusão para este estudo foram ter grau de instrução de no mínimo nível fundamental incompleto, ter sido diagnosticada como estressada; ter um ou mais filho(s) acompanhado(s) neste serviço com diagnóstico de Transtorno do Espectro Autista, não estar em atendimento psicoterápico simultâneo. Os critérios de exclusão foram ter diagnóstico de transtornos psicóticos ou transtornos de personalidade. A amostra geral configurou-se, portanto, como de conveniência, respeitando-se os já citados critérios de inclusão e exclusão.

\section{Material e Instrumentos}

Foram utilizados um Termo de Consentimento Livre e Esclarecido (TCLE), um roteiro de entrevista para mães de crianças com transtornos do espectro autista e o Inventário de Sintomas de Stress para Adultos de Lipp (ISSL). O TCLE foi o documento no qual as mães atestaram o consentimento de sua participação na pesquisa. $\mathrm{O}$ termo 
Moxotó, G. F. A. \& Malagris, L. E. N. (2015). Avaliação de Treino de Controle do Stress para Mães de Crianças com Transtornos do Espectro Autista.

foi lido pela pesquisadora para as participantes, e após a leitura, as mães que desejaram participar do estudo o assinaram. A entrevista possibilitou a coleta de dados sociodemográficos das mães. O ISSL, instrumento elaborado e validado por Lipp (2000) foi utilizado para avaliar presença de stress em fases e sintomatologia predominante física ou psicológica. É composto por três quadros que contêm sintomas físicos e psicológicos de cada fase do stress. O Quadro 1 apresenta os sintomas da $1^{\text {a }}$ fase do stress, o Quadro 2, os sintomas das $2^{\mathrm{a}}$ e $3^{\mathrm{a}}$ fase e o Quadro 3 da quarta fase. O ISSL contém trinta e quatro itens de sintomas físicos e dezenove de sintomas psicológicos.

\section{Procedimentos}

Após aprovação do projeto de pesquisa por Comitê de Ética do Centro de Filosofia e Ciências Humanas da Universidade Federal do Rio de Janeiro, parecer 294.522, foram analisados os dados contidos nos prontuários das mães e de seus filhos para seleção daquelas que atendiam aos critérios de inclusão da pesquisa, configurando assim, uma amostra de conveniência. As selecionadas, depois de serem informadas sobre os objetivos da pesquisa, e concordarem em participar, assinaram o TCLE e responderam as questões do roteiro de entrevista com mães de pessoas com transtornos do espectro autista, e do ISSL. Para a constituição das amostras (experimental e controle) foi utilizado o tipo de amostragem probabilística aleatória simples, na qual cada participante recebeu um número e, posteriormente, foi incluída na amostra que foi submetida a sessões do Treino de Controle do Stress para mães de crianças com transtornos do espectro autista (TCS-MTEA) ou na amostra de controle (AC), a partir de sorteio. Ambas as amostras foram constituídas por 10 mães. A amostra de TCS-MTEA teve 12 sessões individuais com duração de 60 minutos cada e periodicidade semanal. Após o término das sessões, as mães da amostra experimental (AE) responderam ao ISSL. As mães participantes da amostra controle não receberam a intervenção programada na pesquisa, sendo reavaliadas também com o ISSL, da mesma forma que as mães da $\mathrm{AE}$, após o término das sessões do treino, com o objetivo de controlar apenas a passagem do tempo, para servir de parâmetro comparativo com a amostra experimental, ficando em fila de espera para subsequente participação. Após o término do estudo, frente aos resultados favoráveis, foi oferecido às pacientes da $\mathrm{AC}$ o mesmo tratamento recebido pela AE. Foram propostas avaliações de follow up trimestrais e semestrais do stress no Grupo Experimental para verificação da manutenção dos resultados alcançados.

\section{Análise dos Dados}

Para descrever a o perfil da amostra de acordo com as variáveis estudadas foram feitas tabelas de frequência com as variáveis categóricas com valores de frequência absoluta e percentual. Por intermédio da pesquisa foram comparados os seguintes dados, antes e após a intervenção entre a AE de mães submetidas ao Treino de Controle do Stress para mães de pessoas com transtornos do espectro autista (TCS-MTEA) e AC, respectivamente: diagnóstico de stress na $\mathrm{AE}$ e na AC; predominância de tipo de sintoma (físico ou psicológico) na $\mathrm{AE}$ e na $\mathrm{AC}$ e fase do stress em que o paciente se encontra: na AE e na AC. Para a comparação das variáveis categóricas foi utilizado o teste Qui-Quadrado de Pearson, ou o teste exato de Fisher, na presença de valores esperados menores que 5 .

Para a comparação entre as variáveis categóricas pré e pós treino foi utilizado para duas categorias o teste de McNemar e para 3 ou mais categorias o teste de simetria de Bowker. Foi adotado o nível de significância para os testes estatísticos de $5 \%$ ou seja, $p<0,05$.

\section{Resultados}

\section{Roteiro de Entrevista para Mães de Crianças com Transtornos do Espectro Autista}

Em relação aos dados sociodemográficos das mães, quanto à faixa etária, verificou-se que a maioria das participantes tinha entre 31 e 40 anos $(60 \%$ da AE e $70 \%$ da $\mathrm{AC})$, seguidas por aquelas que tinham entre 41 e 50 anos ( $30 \%$ da AE e $20 \%$ da AC) e as que tinham entre 20 e 30 anos ( $10 \%$ de ambas as amostras). Quanto ao grau de instrução, o médio completo foi o mais frequente em ambas as amostras, com seis participantes, sendo três da $\mathrm{AE}(30 \%)$, e três da $\mathrm{AC}(30 \%)$, seguido pelo fundamental completo com cinco participantes, sendo duas da AE (20\%) e três da AC (30\%), superior completo com quatro participantes, sendo três da $\mathrm{AE}(30 \%)$ e uma da $\mathrm{AC}(10 \%)$, médio incompleto com três participantes da $\mathrm{AC}(30 \%)$ e superior incompleto com duas participantes da AE (20\%). Quanto ao estado civil, todas eram casadas (100\%). As 20 participantes eram mães biológicas (100\%). Em ambas as amostras quatro tinham outros filhos (40\%) e as outras seis não $(60 \%)$. Em relação a trabalho, na AE quatro participantes trabalhavam $(40 \%)$ e seis não o faziam (60\%), enquanto na $\mathrm{AC}$, duas $(20 \%)$ participantes trabalhavam e oito não $(80 \%)$.

\section{ISSL}

Stress das Amostras no Pré Treino. Antes da intervenção, quanto às fases do stress, na $\mathrm{AE}$ uma estava em fase de resistência (10\%), enquanto nove estavam em fase de quase exaustão (90\%). Já na AC, cinco participantes estavam em fase de resistência $(50 \%)$ e cinco em fase de quase exaustão (50\%). Não houve diferença significativa entre as amostras quanto à frequência de stress no pré treino, uma vez que estavam todas estressadas (Teste Exato de Fisher, $p$ $=0,141)$. Quanto à sintomatologia apresentada, na AE nove apresentavam sintomas predominantemente psicológicos (90\%), enquanto uma apresentava predominância física $(10 \%)$. Na AC todas tinham sintomas predominantemente sintomas psicológicos, não havendo diferença significativa quanto à frequência de sintomas entre as amostras (Teste Exato de Fisher, $p=1,000$; Tabela 1). 
Tabela 1

Distribuição das Participantes quanto ao Nivel de Stress e Predominância de Sintomas Apresentados no Pré Treino

\begin{tabular}{|c|c|c|c|c|c|}
\hline \multirow[t]{2}{*}{ Variáveis } & \multicolumn{2}{|c|}{ Frequência } & \multicolumn{2}{|c|}{ Porcentagem (\%) } & \multirow[t]{2}{*}{$p$-valor } \\
\hline & $\mathrm{AE}$ & $\mathrm{AC}$ & $\mathrm{AE}$ & $\mathrm{AC}$ & \\
\hline \multicolumn{6}{|l|}{ Fases do stress } \\
\hline Alerta & - & - & - & - & \\
\hline Resistência & 1 & 5 & 10 & 50 & 0,141 \\
\hline Quase-exaustão & 9 & 5 & 90 & 50 & \\
\hline Exaustão & - & - & - & - & \\
\hline \multicolumn{6}{|l|}{ Sintomas } \\
\hline Físicos & 1 & - & 10 & - & \\
\hline Psicológicos & 9 & 10 & 90 & 100 & 1,000 \\
\hline
\end{tabular}

Stress das Amostras no Pós Treino. Quanto às fases do stress, após a intervenção, dentre as dez participantes da AE sete estavam sem stress (70\%) e três estavam em resistência (30\%). Já na AC, dez participantes continuavam estressadas, sendo seis em fase de resistência $(60 \%)$ e quatro em fase de quase exaustão (40\%). Houve diferença significativa entre as amostras quanto à frequência do stress, pois sete participantes da AE estavam sem stress. Das três restantes que continuaram estressadas, duas apresentaram melhora saindo da fase de quase exaustão para resistência e apenas uma manteve-se na mesma fase de antes da intervenção. Já na $\mathrm{AC}$ todas as participantes da AC continuaram estressadas (Teste Exato de Fisher, $p$ $=0,002$; Tabela 2 ). No que se refere às três participantes que continuaram estressadas na $\mathrm{AE}$, cabe ressaltar que uma delas passou por uma crise conjugal, com separação temporária entre a sétima e a nona sessão do treino. Quanto à sintomatologia apresentada, na $\mathrm{AE}$ duas apresentavam sintomas predominantemente psicológicos (20\%), enquanto uma apresentava predominância física $(10 \%)$. $\mathrm{Na} \mathrm{AC}$ todas tinham sintomas predominantemente psicológicos. Houve diferença significativa entre as amostras na frequência de sintomas, pois enquanto as dez da amostra controle apresentavam sintomas, na $\mathrm{AE}$ só três os apresentavam (Teste Exato de Fisher, $p=0,001$ ).

Houve ainda na AE diferença significativa entre as participantes quanto a presença de stress intra amostra nos momentos pré e pós treino, uma vez que após a intervenção sete participantes $(70 \%)$ ficaram sem stress dentre as 10 inicialmente estressadas, resultando em diferença significativa segundo o Teste de McNemar ( $\mathrm{S}=7,00 ; G L$ $=1 ; p=0,008)$.

Além disto, foi verificada redução nas fases do stress dentre as participantes da $\mathrm{AE}$, comparando os momentos pré e pós treino, pois antes do treino nove participantes estavam em quase exaustão e uma em resistência, e após

Tabela 2

Distribuição das Participantes quanto ao Nivel de Stress Apresentado Antes e Após o Treino

\begin{tabular}{lcccccccc}
\hline & \multicolumn{3}{c}{ Pré Treino } & \multicolumn{5}{c}{ Pós Treino } \\
\cline { 2 - 8 } Stress & \multicolumn{2}{c}{ Frequência } & \multicolumn{2}{c}{ Porcentagem } & \multicolumn{2}{c}{ Frequência } & \multicolumn{2}{c}{ Porcentagem } \\
\hline SE & AC & AE & AC & AE & AC & AE & AC \\
Alerta & - & - & - & - & 7 & - & 70 & - \\
Resistência & - & - & - & - & - & - & - & - \\
Q. Exaustão & 1 & 5 & 10 & 50 & 3 & 6 & 30 & 60 \\
Exaustão & 9 & 5 & 90 & 50 & - & 4 & - & 40 \\
Total & - & - & - & - & - & - & - & - \\
\hline
\end{tabular}

Nota. Pós treino $(p=0,002)$. 
Moxotó, G. F. A. \& Malagris, L. E. N. (2015). Avaliação de Treino de Controle do Stress para Mães de Crianças com Transtornos do Espectro Autista.

o treino sete estavam sem stress e três em resistência, configurando diferença significativa na redução de fases de stress segundo Teste de Simetria $(\mathrm{S}=10,00 ; G L=3$; $p=0,019)$.

As 10 participantes da AC que ficaram em fila de espera foram convidadas para participar da intervenção no final da pesquisa, porém oito haviam iniciado psicoterapia e assim, somente duas compareceram para participar do TCS-MTEA, ficando sem stress ao final. Convém acrescentar que as duas participantes não compareceram às doze sessões semanais consecutivamente e estavam se submetendo concomitantemente a sessões de psicoterapia de família. Quanto às avaliações de follow up, somente cinco participantes que haviam ficado sem stress após a intervenção, compareceram para a primeira avaliação de follow up e apenas quatro compareceram à segunda. Verificou-se que todas elas se mantiveram sem stress.

\section{Discussão}

Quanto aos dados demográficos, em relação à faixa etária, a maioria das participantes tinha entre 31 e 40 anos e o grau de instrução mais frequente foi o de ensino médio completo. Em relação ao estado civil, apesar dos dados da literatura internacional sustentarem que o número de divórcios entre os casais com prole é maior durante a infância dos filhos e que este risco é aumentado para os casais que têm filhos com TEA em virtude dos elevados níveis de stress associados (Hartley et al., 2010), todas as participantes deste estudo eram casadas, o que pode ser atribuído a diferenças culturais. Já em relação ao trabalho, a maior parte das mães não trabalhava e as que trabalhavam o faziam em regime parcial, em consonância com os dados encontrados na literatura que descrevem a tendência de mães de crianças com TEA saírem do mercado de trabalho ou a dedicarem-se às atividades laborativas em horário reduzido. Tal como descrito na literatura, esta situação pode contribuir com o agravamento do stress destas mães, uma vez que tendo que abrir mão de suas atividades profissionais ou de reduzir sua carga horária, além de terem uma perda financeira, também podem sentir-se frustradas por não conseguirem dedicar-se ao trabalho que muitas vezes é fonte de satisfação, de elevação de autoestima e de manutenção de autonomia (Cidav et al., 2012).

Quanto aos resultados do ISSL, o fato de terem sido encontrados elevados níveis de stress em ambas as amostras, com nove participantes da $\mathrm{AE}$ e cinco da $\mathrm{AC}$ em fase de quase exaustão e as seis demais em fase de resistência, corrobora os dados da literatura nacional (Bosa, 2002; Semensato, Schmidt, \& Bosa, 2010, Sifuentes \& Bosa, 2010) e internacional (Wang et al., 2013; Zablotsky, Anderson, \& Law, 2013). Concordantemente, diversos autores descrevem as mães de crianças com TEA com altos níveis de stress, mesmo em diferentes contextos culturais e consequentes diferenças nas rotinas vivenciadas (Bilgin \& Kucuk, 2010).
Para compreender a magnitude do agravo sofrido por estas mães pelo stress excessivo, haja vista que a maioria encontrava-se na fase de quase exaustão, é pertinente relembrar que esta fase ocorre quando o indivíduo já não consegue recuperar homeostase interna, não resistindo mais às tensões e assim as defesas do organismo começam a sucumbir. Há alternância de momentos nos quais consegue resistir e sentir-se um pouco melhor, e outros nos quais não consegue mais apresentar resistência. Desta forma, patologias podem começar a surgir, uma vez que a eficácia de resistência já não é mais tão efetiva (Lipp \& Malagris, 2011).

Outras populações que tiveram níveis de stress investigados em diferentes pesquisas apresentaram níveis inferiores aos destas mães com maior prevalência da fase de resistência. Sadir (2010) investigou a eficácia do TCS em relações interpessoais encontrando a maior parcela dos participantes estressados em fase de resistência enquanto a pesquisa de validação do ISSL realizada em diversos estados brasileiros teve $53 \%$ da amostra de estressados encontrando-se em fase de resistência (Lipp, 2000), evidenciando níveis de stress mais alto dentre as mães de filhos com TEA participantes do presente estudo que na população em geral.

Em contrapartida, Schmidt e Bosa (2007) num estudo que investigou níveis de stress em trinta mães de indivíduos de doze a trinta anos com autismo utilizando o ISSL, observaram que $70 \%$ da amostra estava estressada e dentre elas a maioria estava em fase de resistência. É possível que a diferença de faixa etária dos filhos adolescentes e adultos tenha resultado em níveis de stress inferiores ao da amostra estudada neste trabalho, cujos filhos eram crianças. Schmidt e Bosa observaram também, maior frequência de sintomas psicológicos nas mães estressadas tal como na amostra estudada, onde dezenove das vinte mães participantes tinham predominância de sintomas psicológicos.

Quanto à investigação da eficácia do TCS-MTEA, principal objetivo deste estudo, confirmou-se nesta amostra a redução do stress das mães participantes do TCS-MTEA em comparação à AC confirmada pelo fato de que das dez mães estressadas na $\mathrm{AE}$, sete ficaram sem stress após o treino e as três que continuaram estressadas apresentaram redução de stress mudando da fase de quase exaustão na qual se encontravam para a fase de resistência, havendo assim, redução de stress em todas as participantes. Em contrapartida, todas as mães da AC continuaram estressadas configurando diferença significativa entre as amostras.

Cabe enfatizar que as amostras foram comparadas quanto aos níveis de stress das mães participantes antes da intervenção, não tendo sido encontrada diferença significativa. Adicionalmente, houve diferença significativa nos níveis de stress intra amostra comparando os momentos antes e depois da intervenção na amostra experimental.

Foi assim, corroborada nesta amostra, a eficácia desta adaptação do TCS que já havia sido aplicado com êxito em seu formato original a pacientes que apresentavam patologias diversas (Lipp \& Malagris, 2011). Adicional- 
mente, já haviam sido realizadas adaptações bem sucedidas desta intervenção para populações específicas, tal como Sadir (2010) adaptou com sucesso o TCS para relações interpessoais e Bignotto (2010) para a infância.

Cabe ressaltar que as evidências de eficácia podem estar restritas às características singulares desta amostra, haja vista que enquanto as participantes tinham atendimento interdisciplinar completo no mesmo local para suas crianças, outras mães de filhos com TEA, de acordo com a literatura nacional e internacional vivem uma realidade diferente, tendo como fatores associados ao stress, situações diversas, tais como a espera por vaga em programas de atendimento, dificuldades de acesso a profissionais e identificação de tratamento especializado entre outros (Bosa, 2002; Guralnick, 2000, Yeargin-Allsop et al., 2003). É possível que esta situação de fácil acesso ao tratamento, que se configura de grande valia como suporte social, aliada ao suporte profissional interdisciplinar oferecido no serviço de saúde que frequentam, tenham atuado como fatores protetivos ao stress impedindo-as de atingir níveis mais altos e deixando-as simultaneamente mais suscetíveis a responder favoravelmente ao TCS-MTEA. Embora os resultados alcançados não possam ser generalizados devido ao tamanho reduzido da amostra estudada, e tenham que ser interpretados com cautela, acredita-se que possam ser úteis como base para outros estudos com amostras de outras culturas e instituições, para confirmação dos resultados aqui encontrados.

Sugerem-se ainda, estudos de avaliação do TCS-MTEA, comparativos com amostras que recebam outros tipos de intervenção, como psicoterapia individual, familiar ou em grupo e ainda que sejam realizadas avaliações de stress de seguimento com todas as participantes. Assim o TCS-MTEA pode ser aperfeiçoado, proporcionando mais um recurso terapêutico para estas mães, propiciando seu empoderamento, melhorando sua qualidade de vida e de sua família em todas as áreas e contribuindo para o êxito do tratamento de seus filhos com TEA.

\section{Referências}

Antoni, M. H., Pereira, D. B., Marion, I., Ennis, N., Andrasik, M. P., Rose, R., ...O'Sullivan, M. J. (2009). Stress management effects on perceived stress and cervical neoplasia in lowincome HIV-infected women. Journal of Psychosomatic Research, 67(5), 452-463. doi:10.1016/j.jpsychores.2008.06.002

Bellé, A. H., Andreazza, A. C., Ruschel, J., \& Bosa, C. A. (2009). Estresse e adaptação psicossocial em mães de crianças com transtorno de déficit de atenção/hiperatividade. Psicologia: Reflexão e Crítica, 22(3), 317-325. doi:10.1590/S010279722009000300001

Bendixen, R. M., Elder, J. H., Donaldson, S., Kairalla, J. A., Valcante, G., \& Ferdig, R. E. (2011). Effects of a father-based in-home intervention on perceived stress and family dynamics in parents of children with autism. American Journal of Occupational Therapy, 65(6), 679-687.

Bignotto, M. M. (2010). A eficácia do treino de controle do stress infantil (Tese de doutorado em Psicologia não publicada, Pontifícia Universidade Católica de Campinas, SP, Brasil).
Bilgin, H., \& Kucuk, L. (2010). Raising an autistic child: Perspectives from Turkish mothers. Journal of Child and Adolescent Psychiatric Nursing, 23(2), 92-99. doi:10.1111/j.17446171.2010.00228.x

Bosa, C. (2002). Autismo: Atuais interpretações para antigas observações. In C. R. Baptista \& C. Bosa (Eds.), Autismo e educação: Reflexões e propostas de intervenção (pp. 21-39). Porto Alegre, RS: Artmed.

Bougea, A., Darviri, C., \& Alexopoulos, E. C. (2011). A systematic review of randomized controlled interventions for parents' distress in pediatric leukemia. Oncology. doi: $10.5402 / 2011 / 959247$

Bourke-Taylor, H., Pallant, J. F., Law, M., \& Howie, L. (2012). Predicting mental health among mothers of school-aged children with developmental disabilities: The relative contribution of child, maternal and environmental factors. Research in Developmental Disabilities, 33(6), 1732-1740. doi:10.1016/j. ridd.2012.04.011

Bradford, R. (1997). Children, families and chronic disease: Psychological models and methods of care. London: Routledge.

Cadman, T., Eklund, H., Howley, D., Hayward, H., Clarke, H., Findon, J., ...Glaser, K. (2012). Caregiver burden as people with autism spectrum disorder and attention-deficit/hyperactivity disorder transition into adolescence and adulthood in the United Kingdom. Journal of the American Academy of Child and Adolescent Psychiatry, 51(9), 879-888. doi:10.1016/j. jaac.2012.06.017

Cappe, E., Wolff, M., Bobet, R., \& Adrien, J. L. (2011). Quality of life: A key variable to consider in the evaluation of adjustment in parents of children with autism spectrum disorders and in the development of relevant support and assistance programmes. Quality of Life Research, 20(8), 1279-1294. doi:10.1007/s11136-011-9861-3

Carter, B., \& McGoldrick, M. (1995). As mudanças no ciclo de vida familiar: Uma estrutura para a terapia familiar. Porto Alegre, RS: Artmed.

Castro, E. K., \& Piccinini, C. A. (2002). Implicações da doença orgânica crônica na infância para as relações familiares: Algumas questões teóricas. Psicologia: Reflexão e Crítica, 15(3), 625-635.

Cidav, Z., Marcus, S. C., \& Mandell, D. S. (2012). Implications of childhood autism for parental employment and earnings. Pediatrics, 129(4), 617-623. doi:10.1542/peds.2011-2700

Cortez, M. B., \& Souza, L. (2008). Mulheres (in)subordinadas: O empoderamento feminino e suas repercussões nas ocorrências de violência conjugal. Psicologia: Teoria e Pesquisa, 24(2), 171-180.

Dabrowska, A., \& Pisula, E. (2010). Parenting stress and coping styles in mothers and fathers of pre-school children with autism and Down syndrome. Journal of Intellectual Disabilities Research, 54(3), 266-280. doi:10.1111/j.13652788.2010.01258.x

Ellis, A. (1973). Humanistic psychology: The rational emotive approach. New York: Julian.

Fávero, M. A. B., \& Santos, M. A. (2005). Autismo infantil e estresse familiar: Uma revisão sistemática da literatura. Psicologia: Reflexão e Crítica, 18(3), 358-369.

Gomes, V., \& Bosa, C. (2004). Estresse e relações familiares na perspectiva de irmãos de indivíduos com Transtornos Globais do Desenvolvimento. Estudos de Psicologia (Natal), 9(3), 553-561.

Guralnick, M. J. (2000). Early childhood intervention: Evolution of a system. Focus on Autism and Other Developmental Disabilities, 15(2), 68-79. 
Moxotó, G. F. A. \& Malagris, L. E. N. (2015). Avaliação de Treino de Controle do Stress para Mães de Crianças com Transtornos do Espectro Autista.

Hartley, S. L., Barker, E. T., Seltzer, M. M., Floyd, F., Greenberg, J., Orsmond, G., \& Bolt, D. (2010). The relative risk and timing of divorce in families of children with an autism spectrum disorder. Journal of Family Psychology, 24(4), 449-457. doi:10.1037/a0019847

Hayes, S. A., \& Watson, S. L. (2012). The impact of parenting stress: A meta-analysis of studies comparing the experience of parenting stress in parents of children with and without autism spectrum disorder. Journal of Autism and Developmental Disorders, 41(6), 783-795. doi:10.1007/s10803-012-1604-y

Hastings, R., Kovshoff, H., Ward, N., Degli-Espinosa, F., Brown, T., \& Remington, B. (2005). Systems analysis of stress and positive perceptions in mothers and fathers of pre-school children with autism. Journal of Autism and Developmental Disorders, 35(5), 635-644.

Kazdin, A. E., \& Whitley, M. K. (2003). Treatment of parental stress to enhance therapeutic change among children referred for aggressive and antisocial behavior. Journal of Consulting and Clinical Psychology, 71, 504-515.

Keypour, M., Arman, S., \& Maracy, M. R. (2011). The effectiveness of cognitive behavioral stress management training on mental health, social interaction and family function in adolescents of families with one Human Immunodeficiency Virus (HIV) positive member. Journal of Research in Medical Science, 16(6), 741-749.

Konstantareas, M. M., \& Papageorgiou, V. (2006). Effects of temperament, symptom severity and level of functioning on maternal stress in Greek children and youth with ASD. Autism, 10(6), 593-607

Lipp, M. E. N. (1984). Stress e suas implicações. Estudos de Psicologia (Campinas), 3, 5-19.

Lipp, M. E. N. (1996). Stress: Conceitos básicos. In M. E. N. Lipp (Ed.), Pesquisas sobre stress no Brasil: Saúde, ocupações e grupos de risco (pp. 17-31) Campinas, SP: Papirus.

Lipp, M. E. N. (2000). Manual do Inventário de Sintomas de Stress para Adultos de Lipp (ISSL). São Paulo, SP: Casa do Psicólogo.

Lipp, M. E. N., \& Malagris, L. E. N. (2001). O stress emocional e seu tratamento. In B. Rangé (Ed.), Psicoterapias cognitivo-comportamentais: Um diálogo com a Psiquiatria (pp. 475490). Porto Alegre, RS: Artmed.

Lipp, M. E. N., \& Malagris, L. E. N. (2011). Estresse: Aspectos históricos, teóricos e clínicos. In B. Rangé (Ed.), Psicoterapias cognitivo-comportamentais: Um diálogo com a Psiquiatria (pp. 617-632). Porto Alegre, RS: Artmed.

Lovell, B., Moss, M., \& Wetherell, M. (2012). The psychosocial, endocrine and immune consequences of caring for a child with autism or ADHD. Psychoneuroendocrinology, 37(4), 534-542. doi:10.1016/j.psyneuen.2011.08.003

Lutgendorf, S. K., Antoni, M. H., Ironson, G., Starr, K., Costello, N., Zuckerman, M., ...Schneiderman, N. (1998). Changes in cognitive coping skills and social support during cognitive behavioral stress management intervention and distress outcomes in symptomatic human immunodeficiency virus (HIV)-seropositive gay men. Psychosomatic Medicine, 60(2), 204-214.

Malagris, L. E. N. (2009). Frustração In M. E. N. Lipp (Ed.), Sentimentos que causam stress: Como lidar com eles (pp. 27-40). Campinas, SP: Papirus

McCubbin, H., \& Patterson, J. (1983). Family transitions: Adaptation to stress. In H. McCubbin \& C. Figley (Eds.), Stress and the family: Vol. 1. Coping with normative transitions (pp. 5-25). New York: Bruner.

Meichenbaun, D. (1985). Stress inoculation training. New York: Plenum Press
Moes, D., Koegel, R. L., Schreibman, L., \& Loos, L. M. (1992). Stress profiles for mothers and fathers of children with autism. Psychological Reports, 71, 1272-1274.

Navarro, F. R. (2007). La construcción social de la parentalidad y los procesos de vinculación y desvinculación padre-hijo. El papel del mediador familiar. Ciências Psicológicas, 1(2), 119-133.

Osborne, L. A., McHugh, L., Saunders, J., \& Reed, P. (2008). Parenting stress reduces the effectiveness of early teaching interventions for autistic spectrum disorders. Journal of Autism and Developmental Disorders, 38(6), 1092-1103.

Phetrasuwan, S., \& Shandor Miles, M. (2009). Parenting stress in mothers of children with autism spectrum disorders. Journal for Specialists in Pediatric Nursing, 14(3), 157-165. doi:10.1111/j.1744-6155.2009.00188.x

Sadir, M. A. (2010). A influência do treino de controle do stress nas relações de trabalho (Tese de doutorado em Psicologia não publicada, Pontifícia Universidade Católica de Campinas, SP, Brasil).

Sanini, C., Brum, E. H. M., \& Bosa, C. A. (2010). Depressão materna e implicações sobre o desenvolvimento infantil do autismo. Revista Brasileira do Crescimento e Desenvolvimento Humano, 20(3), 809-815.

Schmidt, C. (2004). Estresse, autoeficácia e o contexto de adaptação familiar de mães de portadores de transtorno global do desenvolvimento (Dissertação de mestrado não publicada, Curso de Pós-graduação em Psicologia do Desenvolvimento, Universidade Federal do Rio Grande do Sul, Porto Alegre, RS, Brasil)

Schmidt, C., \& Bosa, C. (2007). Estresse e autoeficácia em mães de pessoas com autismo. Arquivos Brasileiros de Psicologia, 59(2), 179-191.

Semensato, M., Schmidt, C., \& Bosa, C. (2010). Grupo de familiares de pessoas com autismo: Relatos de experiências parentais. Aletheia, 32, 183-194.

Sifuentes, M., \& Bosa, C. A. (2010). Criando pré-escolares com autismo: Características e desafios da coparentalidade. Psicologia em Estudo (Maringá), 15(3), 477-485.

Steinmetz, J. I., Kaplan, R. M., \& Miller, G. L. (1982). Stress management: An assessment questionnaire for evaluating interventions and comparing groups. Journal of Occupational Medicine, 24(11), 923-931.

Szymanski, H. (2004). Práticas educativas familiares: A família como foco de atenção psicoeducacional. Estudos de Psicologia (Campinas), 21(2), 5-16.

Tonge, B., \& Brereton, A. (2011). Autism spectrum disorders. Australian Family Physician, 40(9), 672-677.

Wang, J., Hu, Y., Wang, Y., Qin, X., Xia, W., Sun, C., ...Wang, J. (2013). Parenting stress in Chinese mothers of children with autism spectrum disorders. Psychiatric Epidemiology, 45(1), 135-170. doi:10.1007/s00127-012-0569-7

Yeargin-Allsop, M., Rice, C., Karapukar, T., Doernberg, N., Boyle, C., \& Murphy, C. (2003). Prevalence of autism in a US metropolitan area. JAMA, 289(1), 49-55.

Zablotsky, B., Anderson, C., \& Law, P. J. (2013). The association between child autism symptomatology, maternal quality of life, and risk for depression. Journal of Autism and Developmental Disorders, 43(8), 1946-1955. doi:10.1007/ s10803-012-1745-z

Recebido: 19/12/2014

$1^{a}$ revisão: $24 / 05 / 2015$

Aceite final: $29 / 05 / 2015$ 